\title{
DNA methylation and gene expression dynamics during spermatogonial stem cell differentiation in the early postnatal mouse testis
}

\author{
Naoki Kubo ${ }^{1,2}$, Hidehiro Toh ${ }^{1}$, Kenjiro Shirane ${ }^{1}$, Takayuki Shirakawa ${ }^{3}$, Hisato Kobayashi ${ }^{4}$, Tetsuya Sato ${ }^{5}$, \\ Hidetoshi Sone ${ }^{3}$, Yasuyuki Sato ${ }^{3}$, Shin-ichi Tomizawa ${ }^{3}$, Yoshinori Tsurusaki ${ }^{6}$, Hiroki Shibata ${ }^{7}$, Hirotomo Saitsu ${ }^{6}$, \\ Yutaka Suzuki $^{8}$, Naomichi Matsumoto ${ }^{6}$, Mikita Suyama ${ }^{5}$, Tomohiro Kono ${ }^{4,9}$, Kazuyuki Ohbo ${ }^{3}$ and Hiroyuki Sasaki ${ }^{*}$
}

\begin{abstract}
Background: In the male germline, neonatal prospermatogonia give rise to spermatogonia, which include stem cell population (undifferentiated spermatogonia) that supports continuous spermatogenesis in adults. Although the levels of DNA methyltransferases change dynamically in the neonatal and early postnatal male germ cells, detailed genome-wide DNA methylation profiles of these cells during the stem cell formation and differentiation have not been reported.

Results: To understand the regulation of spermatogonial stem cell formation and differentiation, we examined the DNA methylation and gene expression dynamics of male mouse germ cells at the critical stages: neonatal prospermatogonia, and early postntal (day 7) undifferentiated and differentiating spermatogonia. We found large partially methylated domains similar to those found in cancer cells and placenta in all these germ cells, and high levels of non-CG methylation and 5-hydroxymethylcytosines in neonatal prospermatogonia. Although the global CG methylation levels were stable in early postnatal male germ cells, and despite the reported scarcity of differential methylation in the adult spermatogonial stem cells, we identified many regions showing stage-specific differential methylation in and around genes important for stem cell function and spermatogenesis. These regions contained binding sites for specific transcription factors including the SOX family members.

Conclusions: Our findings show a distinctive and dynamic regulation of DNA methylation during spermatogonial stem cell formation and differentiation in the neonatal and early postnatal testes. Furthermore, we revealed a unique accumulation and distribution of non-CG methylation and $5 \mathrm{hmC}$ marks in neonatal prospermatogonia. These findings contrast with the reported scarcity of differential methylation in adult spermatogonial stem cell differentiation and represent a unique phase of male germ cell development.
\end{abstract}

Keywords: DNA methylation, Non-CG methylation, 5-hydroxymethylcytosine, Spermatogenesis, Spermatogonial stem cell, Prospermatogonia, Spermatogonia

\section{Background}

In mammalian spermatogenesis, huge numbers of spermatozoa are produced throughout adult life. This constant supply is supported by the spermatogonial stem cell (SSC) system [1-3]. In male mouse embryos, primordial germ cells become arrested at the G1/G0 phase of the cell cycle around embryonic day 13 (E13), giving rise to

\footnotetext{
* Correspondence: hsasaki@bioreg.kyushu-u.ac.jp

'Division of Epigenomics and Development, Medical Institute of Bioregulation, Kyushu University, Fukuoka 812-8582, Japan

Full list of author information is available at the end of the article
}

prospermatogonia (PSGs, also known as gonocytes). The PSGs resume mitosis after birth, resulting in the formation of spermatogonia (SGs) [4]. Although the first round of spermatogenesis skips the stem cell stage [5], the mouse testis at postnatal day 7 (P7) already contains both undifferentiated and differentiating SGs $[5,6]$, the former of which are thought to include the initial SSC population [7]. Some cytological markers such as Kit (also known as c-Kit) are available to distinguish between undifferentiated and differentiating SGs [7-12]. Only Kit-negative (Kit $\left.{ }^{-}\right)$SGs contain the SSC 
population (the undifferentiated SGs), and Kit-positive $\left(\mathrm{Kit}^{+}\right)$SGs are the differentiating SGs, where Kit is important for migration, proliferation, and differentiation [13-15]. However, the mechanisms involved in the genetic and epigenetic regulation of SSC formation and differentiation in early postnatal testis are largely unknown.

It is well established that DNA methylation is important for germ cell development [16]. In fetal PSGs, the de novo DNA methyltransferase DNMT3A and its cofactor DNMT3L are expressed highly, and paternally methylated imprinting control regions (ICRs) and many retrotransposons are methylated de novo [17-19]. Thus, neonatal PSGs have a high level of genome-wide methylation. Targeted disruption of the Dnmt3a or Dnmt3l genes in the male germline results in developmental arrest at the spermatocyte stage and subsequent loss of germ cells, indicating an essential role of methylation in spermatogenesis [20, 21]. However, the detailed methylation profile of neonatal PSGs has not been reported. Furthermore, it is totally unknown how DNA methylation and gene expression profiles change during the transitions from PSGs to undifferentiated SGs and from undifferentiated to differentiating SGs in early postnatal testis.

Despite the lack of molecular studies, interesting cytological observations have been made. First, an immunofluorescence study using an anti-5-methylcytosine $(5 \mathrm{mC})$ antibody showed that chromosome arms lose staining in a replication-dependent way upon the transition from neonatal PSGs to SGs [22]. Interestingly, the centromeric regions were barely stained in these cells. Second, increased production of DNMT3A and DNMT3B was observed during the transition from undifferentiated to differentiating SGs in early postnatal and adult testes [23]. It was also shown that undifferentiated SGs were less intensely stained for $5 \mathrm{mC}$ than were differentiating SGs. Thus, there might be an epigenetic switch important for the transition from undifferentiated to differentiating SGs [23]. However, a recent whole-genome bisulfite sequencing (WGBS) study showed that methylation differences are rather rare between undifferentiated $\left(\mathrm{Thyl}^{+}\right)$and differentiating $\left(\mathrm{Kit}^{+}\right)$SGs in adult testis and that only a few promoter regions show differences [24].

Here, we have determined the DNA methylation and gene expression profiles of highly purified neonatal PSGs and early postnatal SGs by WGBS and RNA sequencing (RNA-seq). We used expression of an Oct4-driven transgene as a marker for germ cells, and Kit as a marker for differentiating SGs. Our WGBS revealed unique distributions of $5 \mathrm{mC}$ and 5-hydroxymethylcytosine (5hmC) and transient accumulation of non-CG methylation. Furthermore, we identified genomic regions showing stagespecific changes in CG methylation that were closely associated with genes having roles in stem cell function, cell proliferation, and spermatogenesis. These regions are rich in binding sites for specific transcription factors and likely represent important regulatory elements. Our results provide insights into the epigenetic regulation of SSC formation and differentiation.

\section{Results \\ WGBS and RNA-seq of neonatal PSGs and early postnatal SGs}

PSGs arrested at the G1/G0 phase resume mitosis in the neonatal testis and give rise to undifferentiated and differentiating SGs by the end of the first week $[4,25]$. The differentiation of SGs coincides with the expression of Kit, and only $\mathrm{Kit}^{-}$SGs contain SSCs [13-15]. We isolated germ cells from transgenic mice carrying an Oct4driven enhanced green fluorescent protein (EGFP) gene based on the expression of the transgene (germ cell marker) and expression of endogenous Kit (differentiation marker). The isolated cells were P0.5 PSGs (Oct4$\left.\mathrm{EGFP}^{+}\right)$, and undifferentiated $\left(\mathrm{Oct}_{4}-\mathrm{EGFP}^{+}, \mathrm{Kit}^{-}\right)$and differentiating P7.5 SGs (Oct4-EGFP ${ }^{+}, \mathrm{Kit}^{+}$) (Fig. 1a).

To construct WGBS libraries, we used the postbisulfite adaptor tagging (PBAT) method, which enables amplification-free library construction from a minute amount of DNA [26]. We obtained $>691$ million uniquely mapped reads for each sample, with an average depth of $>15.5 \times$ per strand, or $>31.0 \times$ per DNA molecule, and a CG coverage of 94.3-94.4\% (Additional file 1: Table S1). For each cell type, independent preparations (biological replicates) were studied to confirm the reproducibility $(R>0.953)$. All samples were spiked with unmethylated lambda DNA to calculate the bisulfite conversion efficiency, which always exceeded $99.5 \%$. This conversion rate is sufficient for the analysis of nonCG (or CH) methylation [27]. For gene expression profiling, poly $(\mathrm{A})^{+}$RNAs from replicate samples were analyzed by RNA-seq $(\mathrm{R}>0.981)$. We normally obtained $>83$ million reads for each sample (Additional file 1: Table S1). For comparison, we processed existing bisulfite sequencing data from E16.5 PSGs [15] and RNA-seq data from adult spermatozoa [28], but also performed additional sequencing with our own samples (Additional file 1: Table S1).

\section{Distinctive gene expression signatures}

We first examined the expression of known molecular markers (Additional file 2: Figure S1). We confirmed that Oct4 and Kit showed the expected expression patterns. Genes highly expressed in E16.5 PSGs such as Dnmt3a and Dnmt3l showed consistent expression in P0.5 PSGs, but Nanos2 was downregulated. SSC markers such as Plzf, Gfra1, Ngn3, Id4, and Nanos2 [29] were expressed in P7.5 $\mathrm{Kit}^{-}$SGs and downregulated in $\mathrm{Kit}^{+}$ SGs. Genes involved in the signal transduction pathways 


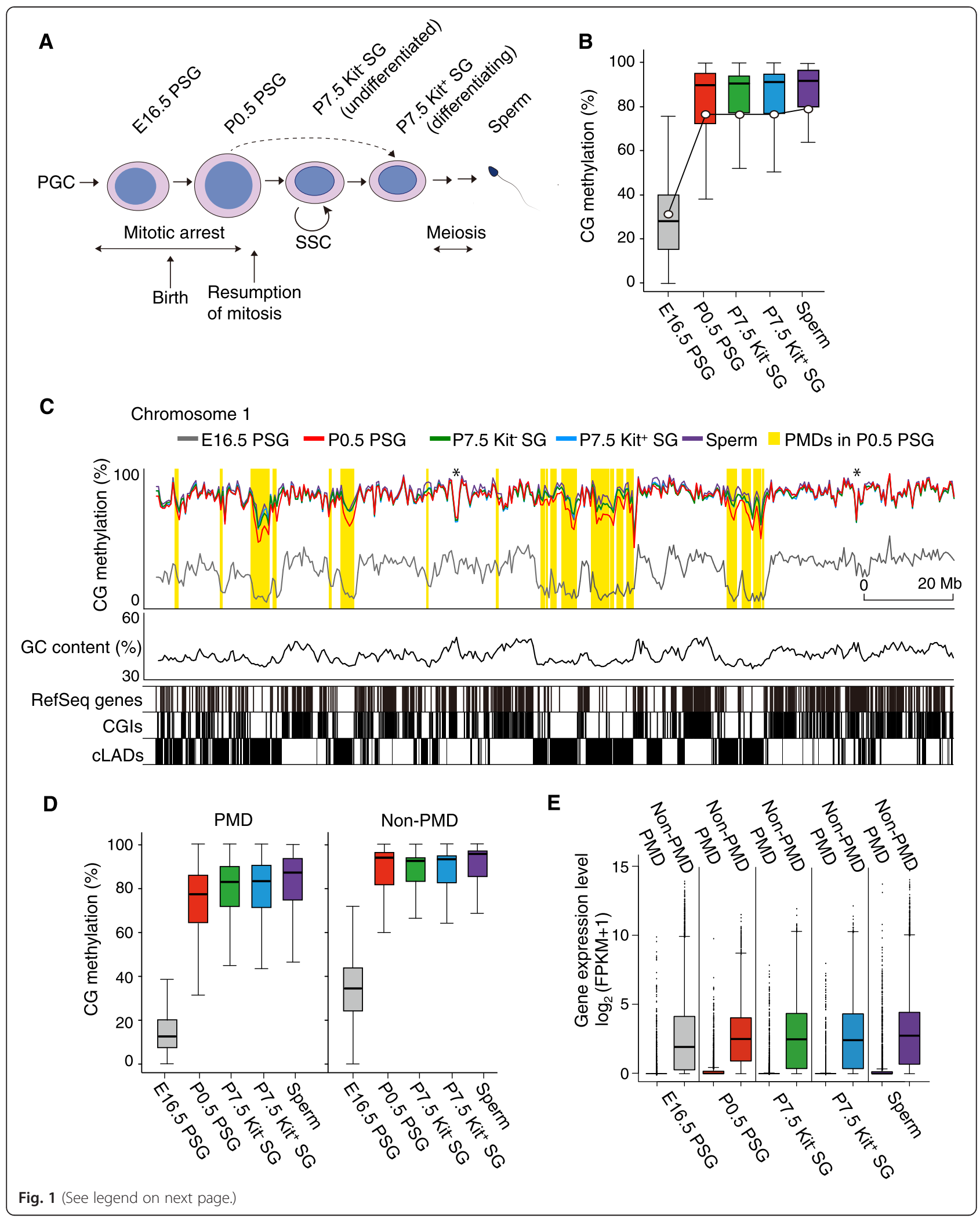


(See figure on previous page.)

Fig. 1 Overall CG methylation levels and distribution. a Schematic representation of male germ cell development and the cell types analyzed in this study. The interrupted arrow shows that the first round of spermatogenesis skips the stem cell stage. PGC, primordial germ cell. $\mathbf{b}$ Developmental changes in the level of CG methylation in the entire genome (line graph) and in $10 \mathrm{~kb}$ windows (box plots). Central bar, median; lower and upper box limits, 25th and 75th percentiles, respectively; whiskers, 1.5 times the interquartile range from the 25th to the 75th percentile. The E16.5 PSG data are from Kobayashi et al. [18]. c Distribution of CG methylation across mouse chromosome 1. The methylation levels determined in 500 kb windows are shown for the respective cell types (colored lines). Large PMDs ( $\geq 500 \mathrm{~kb}$ ) identified in P0.5 PSGs by MethylSeekR are highlighted in yellow. The GC contents in $500 \mathrm{~kb}$ windows are also shown. CLADs are lamina-associated domains common to embryonic stem cells, neural precursor cells, astrocytes, and embryonic fibroblasts [39]. The CG methylation valleys marked with asterisks are not PMDs but composites of unmethylated CGls and hypermethylated segments. $\mathbf{d}$ Developmental changes in the CG methylation level in $10 \mathrm{~kb}$ windows in PMDs and non-PMDs. Those windows overlapping with any PMDs in P0.5 PSGs were considered as PMDs and the rest were considered as non-PMDs. e Transcript levels of genes located in PMDs and non-PMDs. The transcript levels are shown as $\log _{2}$ (FPKM +1 ). The PMD genes are RefSeq coding genes whose promoter regions (from 2000 bp upstream to 500 bp downstream of the transcription start site) overlap with any PMDs. The remaining genes are non-PMD

for SSC self-renewal such as Foxo1, Etv5, Ret, Bcl6b, T (also known as Brachyury), and Cxcr4 [29] were also downregulated in $\mathrm{Kit}^{+}$SGs. Expression of Thy1 was not high in either cell type. In contrast, $\mathrm{Kit}^{+} \mathrm{SGs}$ expressed Stra8, consistent with its role in committing SGs to meiosis. In contrast with our previous cytology finding [23], only Dnmt3b (but not Dnmt3a) was upregulated in $\mathrm{Kit}^{+}$ SGs. All preparations were virtually negative for the Sertoli cell markers Gata4 and Gata6 [30] and the Leydig cell markers Cyp11a1 and Hsd3b1 [31] (Additional file 2: Figure S1). The DNA methylation levels of the germline ICRs [32] also supported negligible somatic contamination. More specifically, in contrast to the $50 \%$ methylation level expected for somatic cells, the paternally methylated ICRs showed high methylation (>80 \%), whereas the maternally methylated ICRs showed low methylation $(<10 \%)$ (Additional file 2: Figure S2A). Overall, our results are consistent with the known distinctive signatures of these prepared cell types.

\section{Large partially methylated domains}

We first compared the overall CG methylation profiles (Fig. 1b). The methylation level increased from $30.1 \%$ in E16.5 PSGs to $76.1 \%$ in P0.5 PSGs, but it did not change much in P7.5 Kit ${ }^{-}$and $\mathrm{Kit}^{+}$SGs $(76.6 \%$ and $76.4 \%$, respectively) (Fig. 1b). The final methylation level in adult spermatozoa was $79.1 \%$. When the methylation level was calculated in nonoverlapping $50 \mathrm{~kb}$ windows, the median value increased consistently from $28.0 \%$ in E16.5 PSGs to $93.9 \%$ in adult spermatozoa, with E16.5 PSGs showing the widest distribution (Fig. 1b).

Interestingly, we found large genomic domains (up to $12.0 \mathrm{Mb}$ ) with relative hypomethylation in all cell types (Fig. 1c; Additional file 2: Figure S3). These domains resembled the partially methylated domains (PMDs) reported in cultured fibroblast cells [33, 34], cancer cells [35, 36], and placenta [37]. The PMDs identified in P0.5 PSGs using MethylSeekR [38] (only those $\geq 500 \mathrm{~kb}$ are shown in Fig. 1c; Additional file 2: Figure S3) were located in genomic regions of low GC content, low CG island (CGI) density, and low gene density, as reported previously for the other cell types. These regions overlapped with constitutive nuclear lamina-associated domains (cLADs) $[35,39]$, which show late DNA replication during the $\mathrm{S}$ phase (Fig. 1c). The existence of PMDs is consistent with the previous observation that whole testis DNA tends to have more hypomethylated CG sites in low GC content regions than in somatic tissues [40]. In addition, some PMDs that we found in $\mathrm{X}$ chromosome were identical to the previously reported large hypomethylated domains or LoDs [41] (Additional file 2: Figure S3).

The PMDs were not present when we examined the WGBS data from E10.5 primordial germ cells, which are undergoing demethylation [18]. The PMDs showed a slow but consistent increase in CG methylation thereafter. In contrast, non-PMD regions obtained high levels of methylation more quickly by P0.5 in PSGs and then changed little (Fig. 1d). Interestingly, previous studies showed that genes in the PMDs are silenced in cancer cells, despite their hypomethylated state [35, 36]. Our RNA-seq results confirmed a similar gene silencing effect at all stages (Fig. 1e). Thus, the PMDs identified in germ cells share not only physical features but also functional consequences with the PMDs reported in other cell types.

\section{High levels of $\mathrm{CH}$ methylation in neonatal PSGs}

We previously reported the presence of $\mathrm{CH}$ (where $\mathrm{H}=$ $\mathrm{A}, \mathrm{C}$, or T) methylation within and around the short interspersed nuclear element (SINE) B1 in neonatal PSGs [42]. The present study revealed surprisingly high levels of genome-wide $\mathrm{CH}$ methylation in P0.5 PSGs. Approximately $10.8 \%$ of all cytosines were methylated in this cell type, compared with 3-4\% in somatic tissues, and more than two-thirds of $5 \mathrm{mCs}$ ( $7.5 \%$ of all cytosines) were at $\mathrm{CH}$ sites (Fig. 2a). Conversely, only one-third of $5 \mathrm{mCs}$ (3.3\% of all cytosines) were at CG sites in P0.5 
A

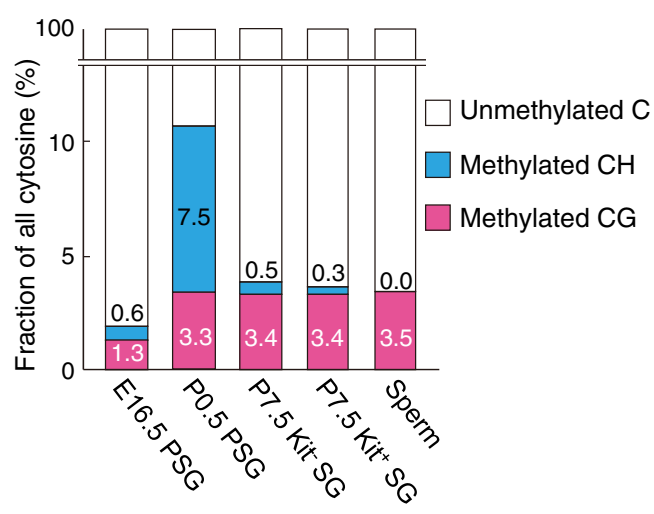

D

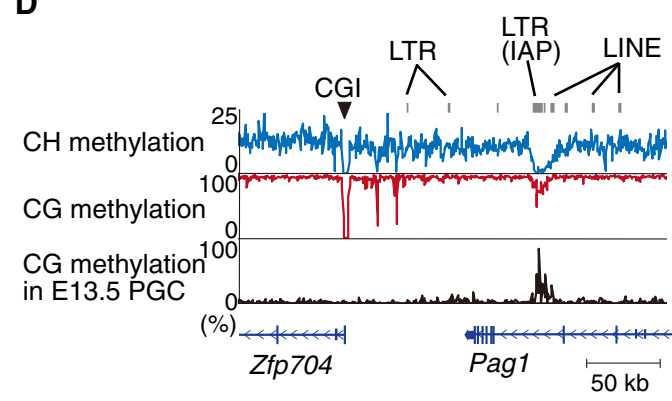

E

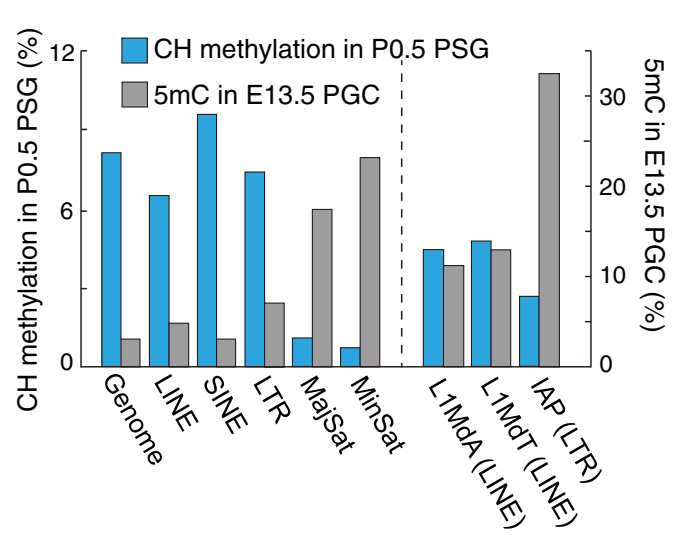

B

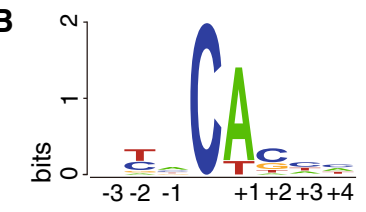

C
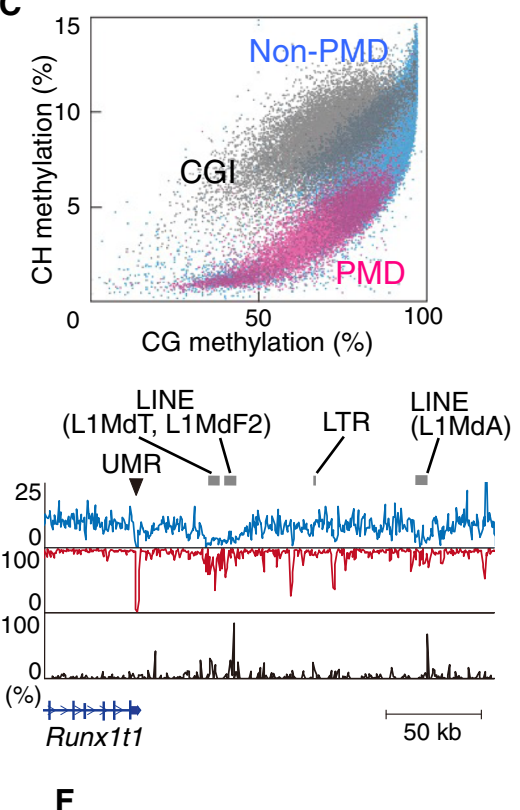

$\mathbf{F}$

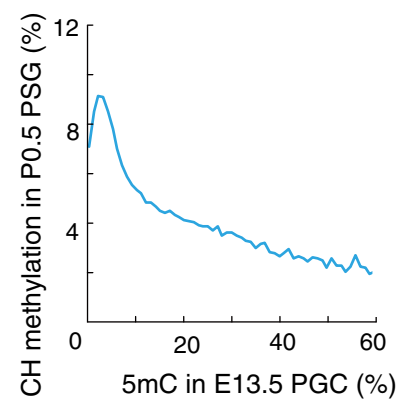

Fig. 2 High levels of CH methylation in neonatal PSGs. a Proportions of methylated cytosines at CG and CH sites, and unmethylated cytosines at all sites. The nonconversion rate was subtracted from the level of methylated cytosine at CG and CH sites, assuming that it occurred randomly at any unmethylated cytosine. b Sequence context of methylated CH in P0.5 PSGs. c A scatter plot showing correlations between the levels of CH and CG methylation in P0.5 PSGs. Each dot represents both levels in a $50 \mathrm{~kb}$ window. The $50 \mathrm{~kb}$ regions were classified into those containing any CGI (almost all of which are non-PMD regions; gray) and those without CGI, and the latter were further classified into PMD (red) or non-PMD regions (blue). d Discrepancy between the CG and CH methylation levels observed at some retrotransposons. The CH (top) and CG methylation (middle) levels in P0.5 PSGs are compared with the CG methylation levels in E13.5 PGCs (bottom) across two genomic regions. An LTR (IAP) (left panel) and LINE copies (right panel) have high levels of CG methylation but very low levels of CH methylation in PSGs. These retrotransposon copies are more CG methylated than the adjacent regions in PGCs. The methylation levels were determined in $500 \mathrm{bp}$ windows. Regions marked with arrowheads are CGl or unmethylated region (UMR). e The CH methylation levels in P0.5 PSGs (blue bar) and the CG methylation levels in E13.5 PGCs (gray bar) in the whole genome and repeat sequences. The levels of the major and minor satellite repeats were calculated from reads aligned to the consensus sequences, while those of the other sequences were calculated from reads uniquely aligned to the reference mouse genome $(\mathrm{mm} 10)$. $\mathbf{f}$ Negative correlation between the $\mathrm{CH}$ methylation levels in P0.5 PSGs and the CG methylation levels in E13.5 PGCs. The methylation levels were calculated in 5 kb windows

PSGs. Among the $\mathrm{CH}$ sequences, CA was the most frequently methylated (methylation level, $16.9 \%$ ) (Fig. 2b). Overall, genomic regions showing high levels of CG methylation (non-PMD regions) had high levels of $\mathrm{CH}$ methylation. Among the non-PMD regions, CGIcontaining regions showed a relatively strong correlation 
between the two levels, but the rest of the non-PMD regions had high $\mathrm{CH}$ methylation only when CG methylation was very high (Fig. 2c).

The typical CGIs were devoid of both CG and $\mathrm{CH}$ methylation (Fig. 2d). Interestingly, some long interspersed nuclear element (LINE) and long terminal repeat (LTR) retrotransposon copies had low levels of $\mathrm{CH}$ methylation, but relatively high levels of CG methylation, with the adjacent regions having high levels of both CG and $\mathrm{CH}$ methylation (Fig. 2d). The LINE copies with low $\mathrm{CH}$ methylation levels belonged to the evolutionarily younger subfamilies of the L1 family, such as L1MdA and L1MdT (Fig. 2e). Likewise, many of the LTR copies devoid of $\mathrm{CH}$ methylation were members of the intracisternal A particle (IAP) family (Fig. 2e), which is again a relatively young class of endogenous retrovirus. It is known that, although the majority of retrotransposons become hypomethylated in primordial germ cells, certain copies stay CG methylated [19], indicating a resistance to reprogramming. Interestingly, the abovementioned LINE and LTR copies, which were devoid of $\mathrm{CH}$ methylation, maintained some level of CG methylation in E13.5 primordial germ cells [18] (Fig. 2d-f). Thus, the de novo DNMTs responsible for $\mathrm{CH}$ methylation in fact introduce fewer $\mathrm{CH}$ methylation marks in regions maintaining residual CG methylation.

\section{$\mathrm{CH}$ methylation explains cytological observations}

A previous immunocytological study showed that the chromosome arms of neonatal PSGs are strongly stained for $5 \mathrm{mC}$ [22] (Additional file 2: Figure S4). Very interestingly, the same study showed that the signals are then lost in a replication-dependent way in early postnatal SGs. Our findings now suggest that the observed signal loss might be caused by the loss of $\mathrm{CH}$ methylation, but not of CG methylation, because the $\mathrm{CH}$ methylation level is more than 2-fold higher than the CG methylation level in neonatal PSGs (Fig. 2a) and there is no maintenance mechanism for $\mathrm{CH}$ methylation in mammals. Indeed, $\mathrm{CH}$ methylation was lost rapidly after the resumption of mitosis (Fig. 2a). Despite the genomewide loss of $\mathrm{CH}$ methylation, the CG methylation levels did not change much in the postnatal SGs (Figs. 1b and 2a). More specifically, high levels of CG methylation (>80 \%) were maintained at the H19, Rasgrf1, and Dlk1Gtl2 ICRs in P7.5 $\mathrm{Kit}^{-}$and $\mathrm{Kit}^{+}$SGs (Additional file 2: Figure S2B). Retrotransposons also maintained high levels of CG methylation, although satellite repeats showed lower methylation levels (Additional file 2: Figure S2C). Consistent with the CG methylation maintenance, genes coding for proteins involved in methylation maintenance, such as Dnmt1 and Uhrf1, were expressed in these cells (Additional file 2: Figure S2D).
High levels of $5 \mathrm{hmC}$ in satellite repeats of neonatal PSGs

$5 \mathrm{hmC}$ has been recognized as an important intermediate for demethylation [43], and its levels and distribution change during spermatogenesis [44]. The abovementioned cytological study showed that the centromeric and pericentromeric regions are only weakly stained for $5 \mathrm{mC}$ in neonatal PSGs and early postnatal SGs [22] (Additional file 2: Figure S4). This was attributed to hydroxylation of $5 \mathrm{mCs}$ in recent studies using anti-5hmC antibodies [45, 46] (Additional file 2: Figure S4). To test this at the molecular level, the $5 \mathrm{hmC}$ profile of P0.5 PSGs was determined by oxidative bisulfite sequencing [47] combined with the PBAT method. Almost all $5 \mathrm{hmCs}$ occurred at CG sites, consistent with a previous study on embryonic stem cells [48], and the genomewide $5 \mathrm{hmC}$ level was $1.4 \%$ (Fig. 3a). As expected, the minor and major satellite repeats, corresponding to the centromeric and pericentromeric regions, respectively, showed higher $5 \mathrm{hmC}$ levels $(7.5 \%$ and $4.8 \%$, respectively) than the whole genome (Fig. 3a). When the frequency of the modified base per length was determined, the major satellites showed the highest $5 \mathrm{hmC}$ density, whereas the whole genome showed the highest $5 \mathrm{mC}$ density (Fig. 3b), consistent with the immunocytological observations. The high level of $5 \mathrm{hmC}$ at the satellite repeats might be important for silencing these repeats in PSGs [46] and, after this stage, the $5 \mathrm{hmC}$ signals disappear in a replication-dependent way $[45,46]$ (Additional file 2: Figure S4). Among the retrotransposons, the IAP family and the younger subfamilies of L1 had high levels of $5 \mathrm{hmC}$ (Fig. 3c). As mentioned above, these retrotransposon species are resistant to reprogramming and retain some levels of CG methylation in E13.5 primordial germ cells (Fig. 3c and d).

\section{Stage-specific differentially methylated regions (DMRs)}

We next sought to identify regions showing developmental stage-specific changes in CG methylation because celltype-specific local hypomethylation often marks active regulatory regions [49]. The CG methylation levels were compared in 500 bp windows ( $\geq 5$ CG sites, 100 bp sliding steps) between P0.5 PSGs and P7.5 Kit ${ }^{-}$or between P7.5 $\mathrm{Kit}^{-}$and $\mathrm{Kit}^{+}$SGs, and regions spanned by overlapping windows showing $>30 \%$ CG methylation differences in either sample pair were identified. As a result, we found a total of 5387 DMRs $(500-2400 \mathrm{bp})(P<0.02$, false discovery rate $(\mathrm{FDR})<0.05)$, which together constituted $0.14 \%$ of the genome. Thus, despite the reported scarcity of methylation differences between undifferentiated $\left(\right.$ Thy $1^{+}$) and differentiating $\left(\mathrm{Kit}^{+}\right.$) adult SGs [24], we identified many DMRs between the undifferentiated and differentiating early postnatal SGs (see below). On the other hand, our DMRs did not include any of the seven promoters 
A

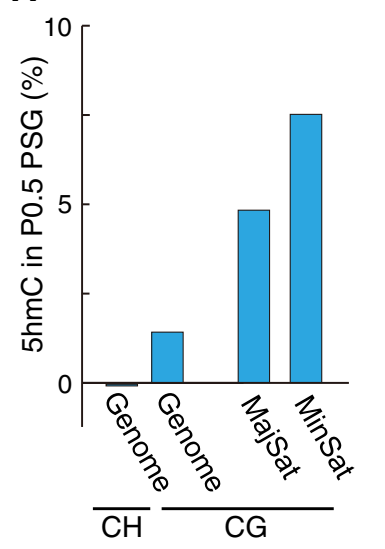

C

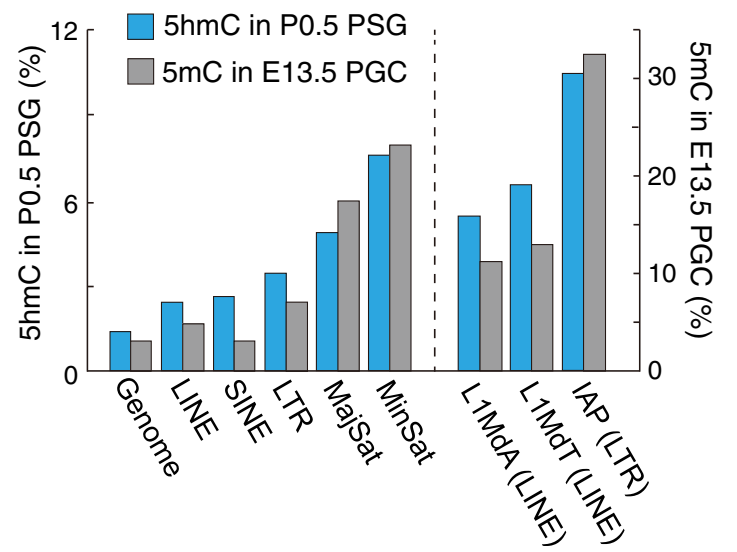

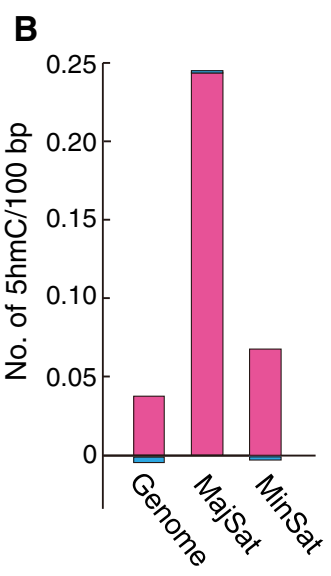

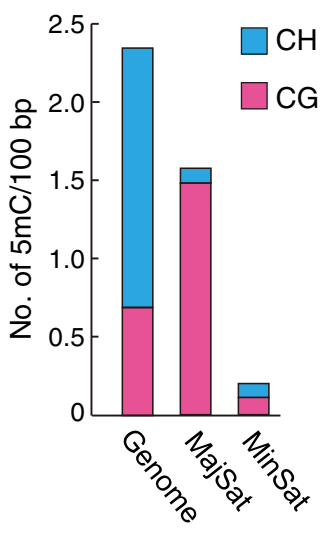

D

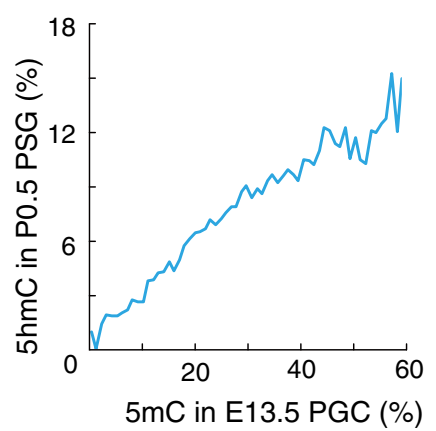

Fig. 3 Distribution of 5hmC in P0.5 PSGs. a 5hmC levels in the whole genome and satellite repeats. The levels in the major and minor satellite repeats were calculated from sequence reads aligned to the consensus sequences. The levels in the whole genome were calculated from reads uniquely aligned to the reference mouse genome ( $\mathrm{mm} 10)$. b Densities of $5 \mathrm{hmC}$ and $5 \mathrm{mC}$ (per $100 \mathrm{bp}$ ) in the whole genome and major and minor satellite repeats. The proportions of $5 \mathrm{hmCs} / 5 \mathrm{mCs}$ at CG and CH sites are also shown. $\mathbf{c}$ The $5 \mathrm{hmC}$ levels of the whole genome and repeat sequence in P0.5 PSGs (blue bar) and the $5 \mathrm{mC}$ levels of those in E13.5 PGCs (gray bar). The levels of the satellite repeats were calculated from reads aligned to the consensus sequences, while those of the other sequences were calculated from reads uniquely aligned to the reference mouse genome (mm10). d Positive correlation between the 5hmC levels in P0.5 PSGs and the $5 \mathrm{mC}$ levels in E13.5 PGCs. The levels were calculated in $5 \mathrm{~kb}$ windows

showing $>30 \%$ CG methylation difference between the undifferentiated and differentiating adult SGs [24].

The DMRs were then subjected to cluster analysis based on the patterns of stage-dependent methylation changes. Six distinct clusters were noted (Fig. 4a; Additional file 3: Table S2). The cluster-1 DMRs $(n=695)$ were highly methylated in P0.5 PSGs and P7.5 Kit ${ }^{-}$SGs but less methylated in $\mathrm{Kit}^{+}$SGs. Cluster-2 DMRs $(n=2093)$ had high levels of methylation in P0.5 PSGs, but showed decreased methylation in P7.5 $\mathrm{Kit}^{-}$SGs and even lower methylation in $\mathrm{Kit}^{+}$SGs. Clusters 5 and $6(n=1316$ and 1097, respectively) showed low methylation in P0.5 PSGs and higher methylation in P7.5 $\mathrm{Kit}^{+}$SGs, but only cluster 5 showed an increase in $\mathrm{Kit}^{-}$SGs. Clusters 3 and 4 had distinct methylation patterns, with cluster-3 gaining methylation during the transition from $\mathrm{Kit}^{-} \mathrm{SG}$ to $\mathrm{Kit}^{+} \mathrm{SGs}$, but there were only small numbers of DMRs in these clusters.

Only a small fraction of the DMRs of each cluster was found to overlap with promoters (Fig. 4b), as reported in undifferentiated and differentiating adult SGs [24]. Rather, most DMRs-especially those belonging to clusters 1 and 2-were intergenic or intronic. When published histone modification data from the adult testis [50] were analyzed, the DMRs of clusters 1 and 2 showed enrichment in enhancer marks such as histone H3 lysine-4 monomethylation (H3K4me1) and histone H3 lysine-27 acetylation (H3K27ac) [51, 52] (Fig. 4c), suggesting that they are distal or intronic regulatory regions. However, in clusters 5 and 6, many of the DMRs were mapped to the PMDs (Fig. 4b). Because the genes in PMDs are 
A

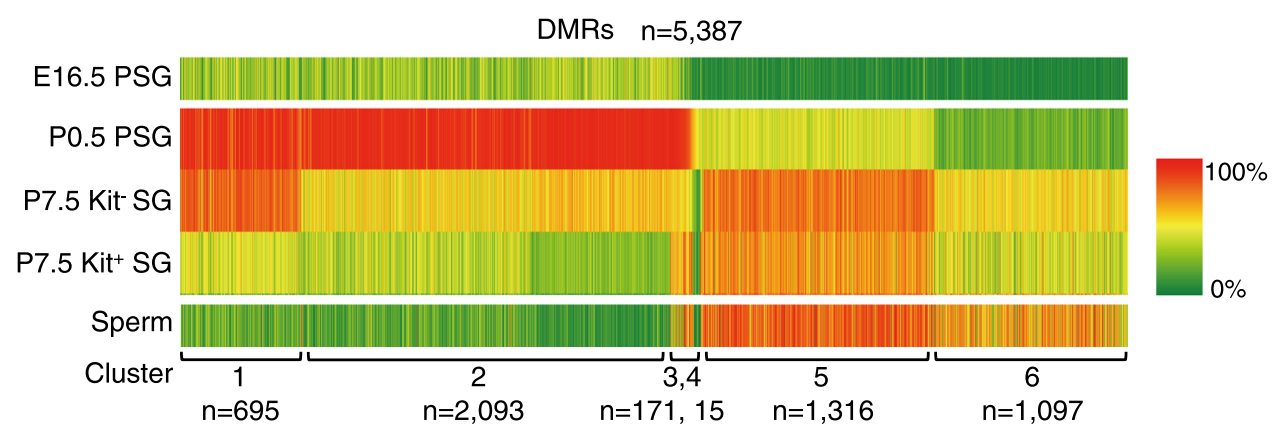

B

Cluster 1 Cluster 2

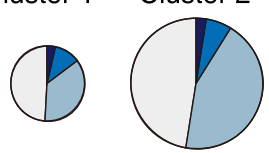

Cluster 3,4

Cluster 5 Cluster 6
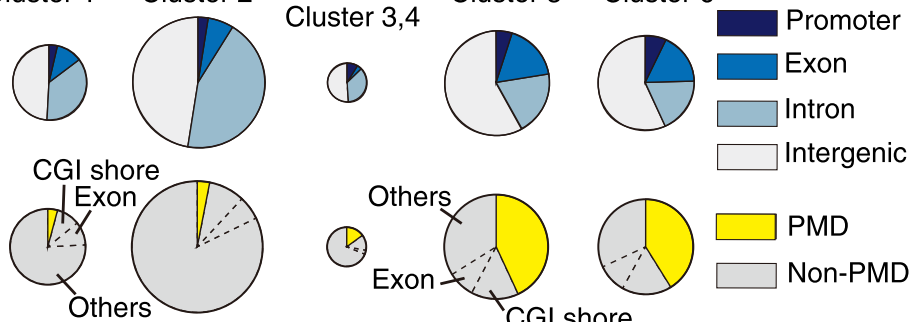

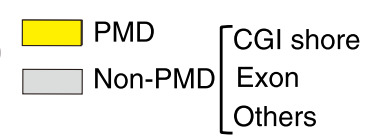

C

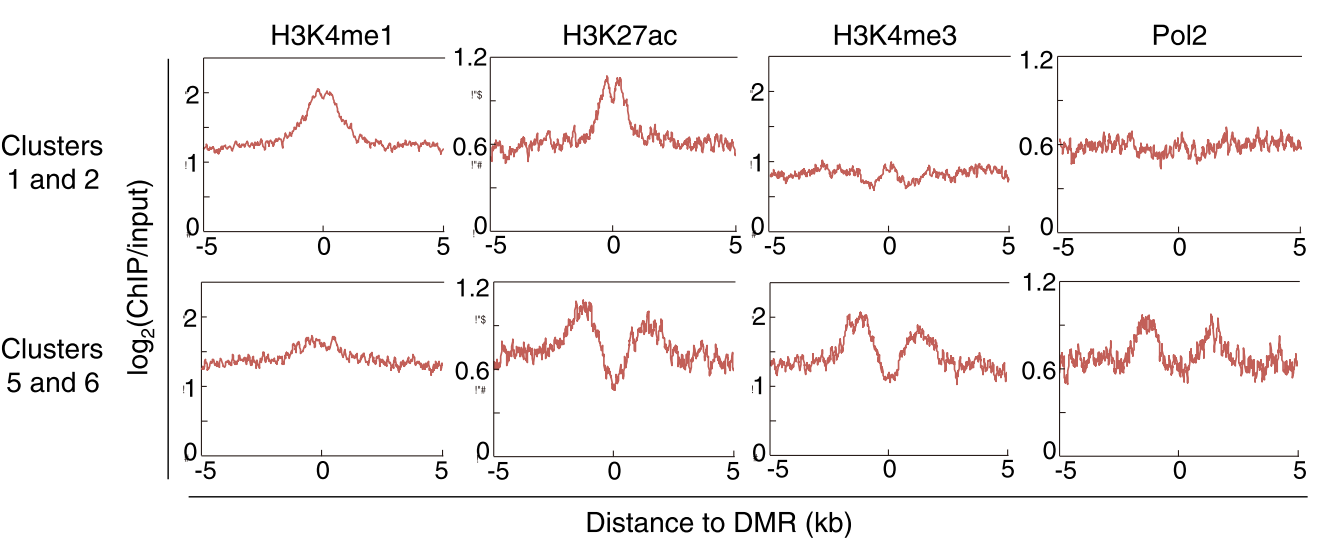

D

Cluster $1 \mathrm{GO}$ biological process terms

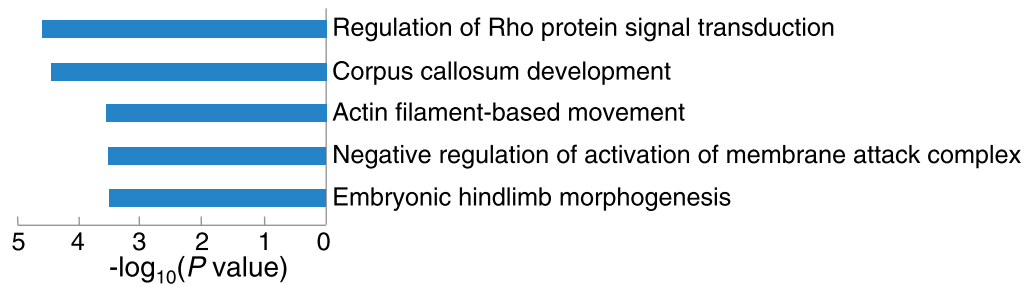

Cluster 1 Mouse phenotype terms

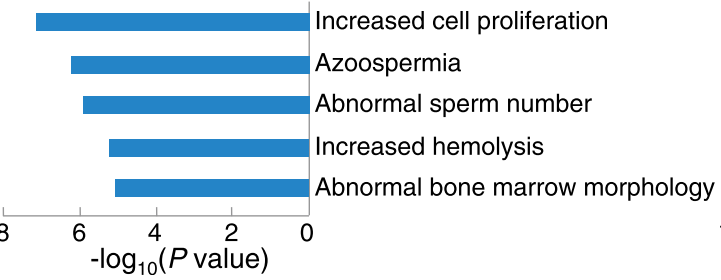

Cluster 2 GO biological process terms

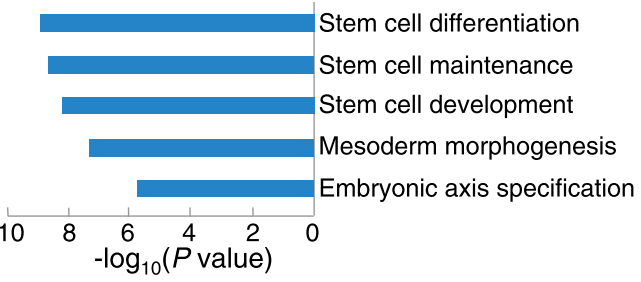

Fig. 4 (See legend on next page.) 
(See figure on previous page.)

Fig. 4 Identification and characterization of stage-specific DMRs. a A heat-map representation of the changes in CG methylation at the DMRs identified in the transitions from P0.5 PSGs to P7.5 Kit ${ }^{-}$SGs and from P7.5 Kit ${ }^{-}$SGs to Kit ${ }^{+}$SGs. The color gradation (from green to red, with an intermediate in yellow) shows the methylation levels (from low to high). The six DMR groups identified by cluster analysis using the changing methylation patterns are shown. The methylation levels in E16.5 PSGs and mature spermatozoa are also shown for comparison. $\mathbf{b}$ Genomic locations of the DMRs of each cluster relative to the gene structure (top) or PMD/non-PMD regions (bottom). The DMRs in non-PMD regions are further subdivided based on whether they overlap with CGI shores (2 kb from the edge of a CGl) or exons. The circle size represents the number of DMRs. c Levels of H3K4me1, H3K4me3, H3K27ac, and RNA polymerase II (Pol2) relative to the DMRs in the adult mouse testis [50]. The data are shown for combined cluster-1 and -2 DMRs and combined cluster-5 and -6 DMRs. $\mathbf{d}$ GO biological process and mouse phenotype terms enriched for the DMRs of clusters 1 and 2 by GREAT analysis

mostly silent through the stages (Fig. 1e), the observed methylation changes did not seem to be relevant to regulation, but might rather reflect the ongoing de novo methylation of PMDs (Fig. 1c and d). The rest of the DMRs of clusters 5 and 6 coincided with CGI shores or exons in non-PMD regions, of which methylation can be a cause or a consequence of gene activity. However, the DMRs of these clusters lacked histone modification marks for enhancers or promoters (Fig. 4c).

We then sought to correlate the DMRs with specific gene functions based on their genomic locations using Genomic Regions Enrichment of Annotations Tool (GREAT) analysis [53]. This revealed that the DMRs of cluster 1 were significantly correlated with gene ontology (GO) biological processes terms "regulation of Rho protein signal transduction" $\left(P=2.5 \times 10^{-5}\right)$ and "actin filament-based movement" $\left(P=2.4 \times 10^{-4}\right) \quad$ (Fig. $4 \mathrm{~d}$; Additional file 4: Table S3), consistent with the active proliferation and movement of differentiating SGs. The DMRs were also associated with mouse phenotype GO terms "cell proliferation" $\left(P=6.6 \times 10^{-8}\right)$, "azoospermia" $\left(P=6.0 \times 10^{-7}\right)$, and "abnormal sperm number" $(P=$ $1.2 \times 10^{-6}$ ) (Fig. 4d; Additional file 4: Table S3). The DMRs of cluster 2 were significantly correlated with the biological process terms "stem cell differentiation" $(P=$ $\left.1.1 \times 10^{-9}\right)$, "stem cell maintenance" $\left(P=2.1 \times 10^{-9}\right)$, and "stem cell development" $\left(P=5.7 \times 10^{-9}\right)$ (Fig. $4 \mathrm{~d}$; Additional file 4: Table S3), consistent with a role in undifferentiated SGs. In contrast, clusters 5 and 6 did not show significant enrichment $(F D R<0.1)$ even though the DMR numbers were sufficient for the analysis.

\section{Correlation between DNA methylation and gene expression profiles}

To correlate the DMRs with gene expression, we next identified genes showing $>2.0$-fold expression changes with absolute difference $(\Delta)$ values in fragments per $\mathrm{kb}$ of transcript per million mapped reads (FPKM) > 5.0. We found 1865 genes upregulated and 893 genes downregulated in P7.5 $\mathrm{Kit}^{-}$SGs compared with P0.5 PSGs (Additional file 2: Figure S5; Additional file 5: Table S4). Also, 135 genes were upregulated and 341 genes downregulated in P7.5 $\mathrm{Kit}^{+}$SGs compared with $\mathrm{Kit}^{-}$SGs (Additional file 2: Figure S5; Additional file 5: Table S4).
On average, the expression changes observed between P0.5 PSGs and P7.5 $\mathrm{Kit}^{-}$SGs were greater than those between $\mathrm{Kit}^{-}$SGs and $\mathrm{Kit}^{+}$SGs. A GO analysis of each set of differentially expressed genes using the Database for Annotation, Visualization and Integrated Discovery (DAVID) tool [54] identified terms such as "cell cycle" and "transmembrane receptor protein tyrosine kinase signaling pathway" (Additional file 6: Table S5).

Because many of the DMRs of clusters 1 and 2 are likely to be regulatory regions (see above), we next sought to correlate these DMRs with gene expression changes. Of the 1150 genes physically linked to the cluster-1 DMRs (identified by GREAT), 182 showed expression changes (>2.0-fold and $\triangle$ FPKM $>5.0$ ) (99 up and 83 down) between P0.5 PSGs and P7.5 Kit ${ }^{-}$SGs, and 64 (17 up and 47 down) between P7.5 Kit ${ }^{-}$and $\mathrm{Kit}^{+}$SGs (Additional file 2: Figure S5). Of the 2772 genes linked to the cluster-2 DMRs, 457 showed expression changes (245 up and 212 down) between P0.5 PSGs and P7.5 Kit ${ }^{-}$SGs, and 162 (14 up and 148 down) between P7.5 $\mathrm{Kit}^{-}$and $\mathrm{Kit}^{+} \mathrm{SGs}$ (Additional file 2: Figure S5). Overall, the probability of showing an expression change was significantly greater for the DMR-linked genes than for the entire gene set $\left(P<1.0 \times 10^{-6}\right.$ by chi-squared test $)$.

Examples of the DMRs with the methylation changes of the genomic region and expression changes of the linked gene are shown in Fig. 5. Stra8 is inducible by retinoic acid and important for the transition into meiosis $[55,56]$. This gene had a cluster-1 DMR in the upstream region, consistent with its highest expression in P7.5 Kit + SGs, and the DMR had high levels of the enhancer histone marks such as H3K4me1 and H3K27ac in adult testis (Fig. 5), suggesting that the DMR has a regulatory function. Plzf encodes a transcriptional regulator required for SSC maintenance [8]. This gene had a cluster1 and a cluster-2 DMR in the upstream region, the latter of which is consistent with its strongest induction in $\mathrm{Kit}^{-}$ SGs. Many other genes involved in the signal transduction pathways for SSC self-renewal (Foxo1, Ret, T, and Cxcr4) also had DMRs of clusters 1 and 2 within or around the genes (within 50-kb upstream and 50-kb downstream) and showed high levels of gene expression in $\mathrm{Kit}^{-} \mathrm{SGs}$ (Additional file 2: Figures S1 and S6). Again, the enhancer marks were found in the DMRs. 


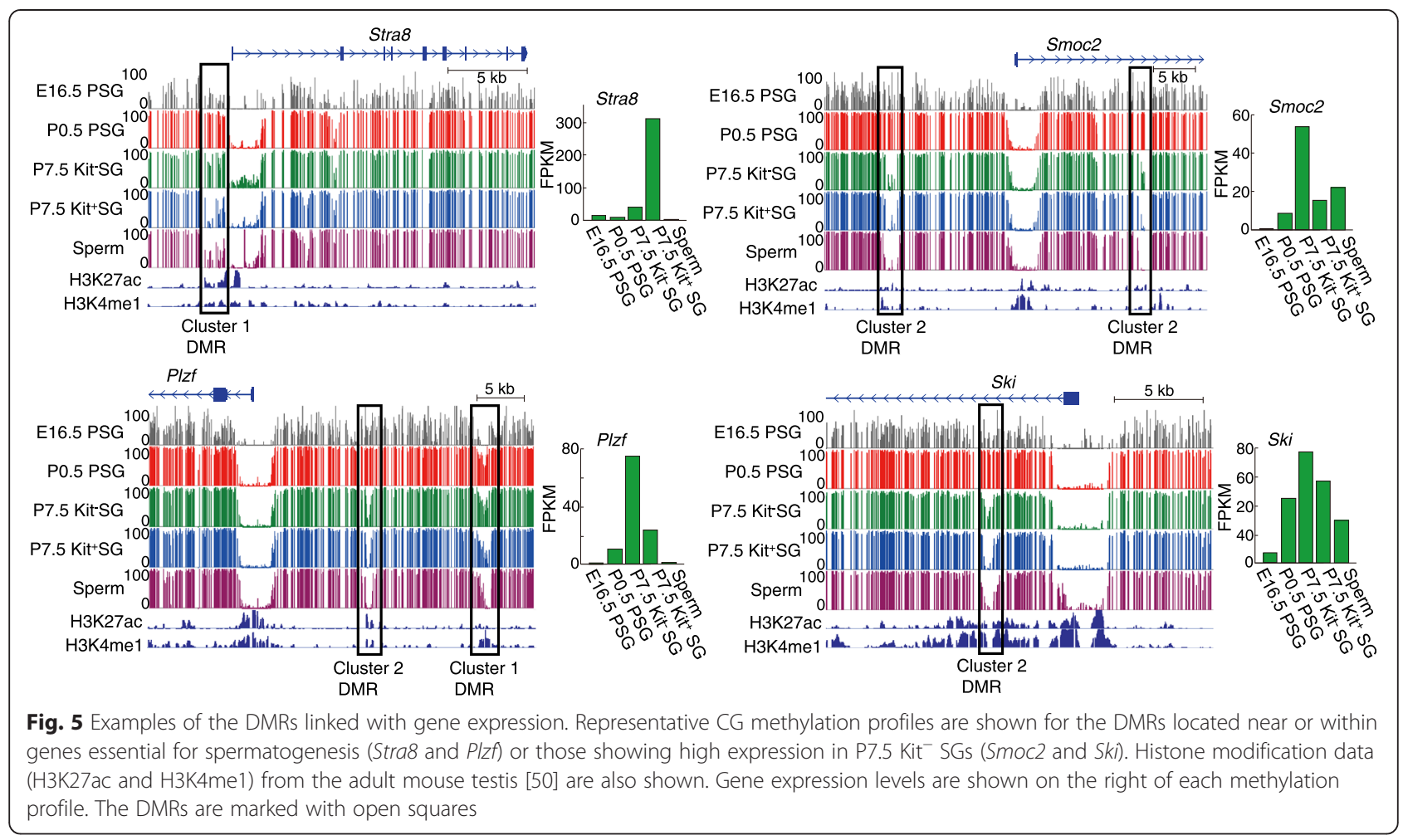

There were also genes showing high levels of expression in P7.5 Kit ${ }^{-}$SGs, but with no reported role so far in SSCs or during spermatogenesis (Fig. 5). Smoc2 encodes a member of the SPARC family matricellular calciumbinding proteins and is highly expressed during embryogenesis and wound healing [57]. This gene had two cluster-2 DMRs, one in the upstream region and the other in an intron, and showed highest expression in Kit - SGs, consistent with its possible role in stem cells [58]. $S k i$ is a proto-oncogene encoding a repressor of transforming growth factor beta activity [59]. This gene had a cluster-2 DMR in an intron and showed expression throughout male germ cell development, with its highest expression in $\mathrm{Kit}^{-} \mathrm{SGs}$. Because Ski expression precedes demethylation of the DMR, its changed methylation status might be secondary. Nevertheless, these results suggest that the combined use of WGBS and RNA-seq can detect potentially important genes and regulatory regions. Interestingly, most DMRs of clusters 1 and 2 remained hypomethylated in the subsequent stages irrespective of gene activity, leaving the epigenetic signatures in the sperm genome (Figs. 4a and 5).

We previously showed that forced expression of Dnmt3b induces Kit expression and that Uhrf1/Np95 (involved in maintenance methylation) ablation interferes with the Kit- SG to Kit + SG transition [23]. This suggests that DNA methylation is important for this transition. Thus cluster-3 DMRs, which gain methylation in Kit + SGs, may be of interest. We therefore looked for genes that are associated with cluster-3 DMRs and show a more than 2-fold expression change in this transition. As a result, three genes were identified (Col22a1, Gfra1, and Pcp4l1), among which Gfra1 encoding a component of the GDNF receptor essential for SSC maintenance (Additional file 2: Figure S6) showed eleven-fold upregulation. The cluster-3 DMR of Grfa1 was located 40-kb downstream of its $3^{\prime}$ end and contained some evolutionarily conserved sequences, suggesting a regulatory role. This may be consistent with the previously observed upregulation of Gfral in Dnmt3l knockout SSCs [60] although it is not clear whether this dysregulation involves impaired methylation.

\section{The DMRs are Rich in binding motifs for specific transcription factors}

Finally, we sought to identify transcription factor binding motifs in the DMR sequences of clusters 1 and 2 by the Hypergeometric Optimization of Motif Enrichment (HOMER) analysis [61]. Short sequence motifs enriched in the DMRs of these clusters were first identified, and known transcription factor binding motifs similar to these "de novo motifs" were discovered. We found that the identified motifs were highly similar to the binding motifs for SOX family members such as SOX10 and SOX3 (Fig. 6a). The critical function of SOX3 in SG differentiation has been established [62]. In contrast, the role of SOX10 is not known for SSCs or in spermatogenesis although it does have a role in the maintenance 


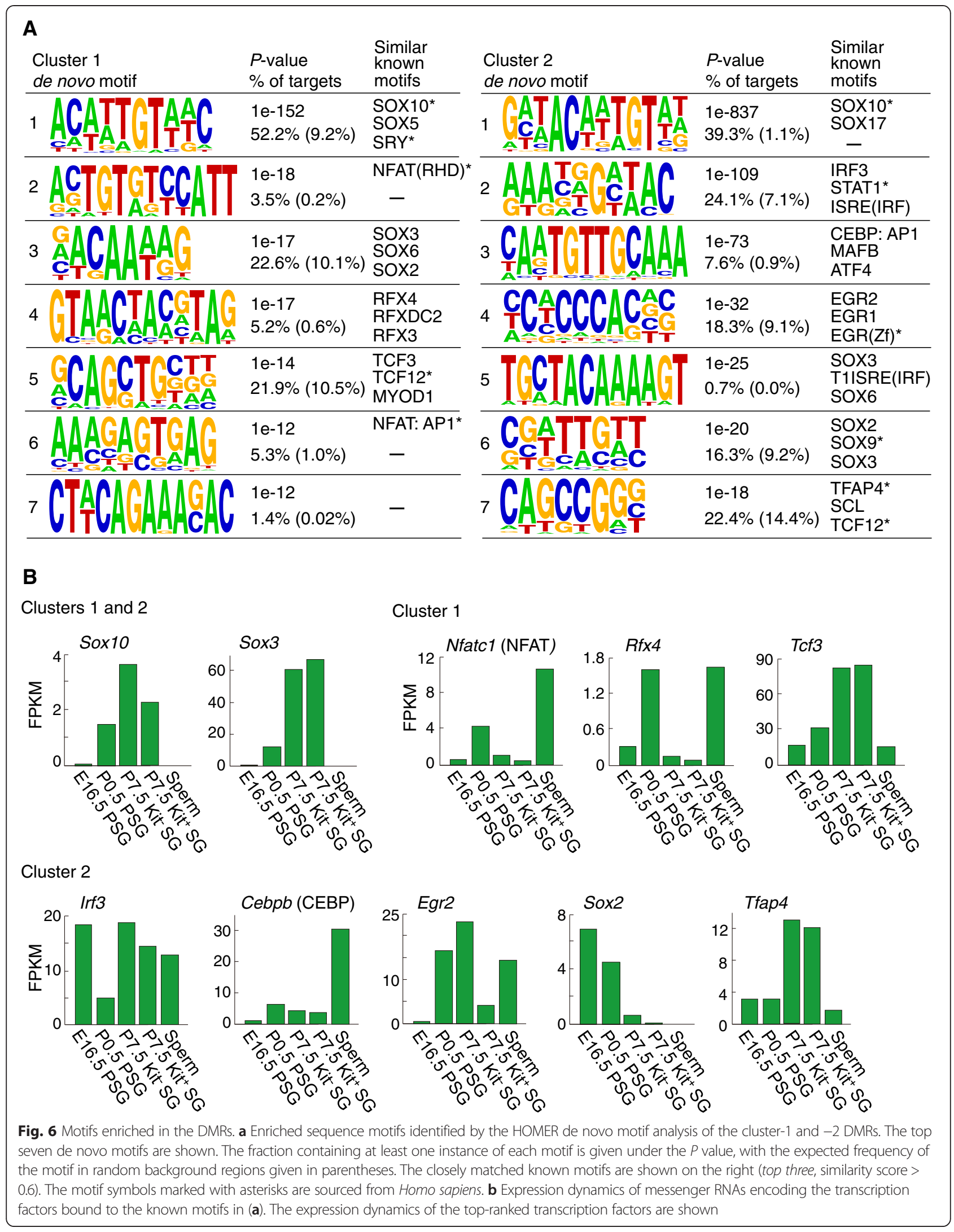


of neural crest stem cells and the melanocyte lineage $[63,64]$. The RNA-seq data showed that Sox3 was highly expressed in P7.5 $\mathrm{Kit}^{-}$and $\mathrm{Kit}^{+}$SGs and that Sox10 was also expressed at lower levels (Fig. 6b).

In addition to the SOX family members, the cluster-1 DMRs showed enrichment in motifs similar to the binding motifs for transcription factors NFATC1 (cytokine induction), RFX4 (transactivation), and TCF3 (also known as E2A) (morphogenesis) (Fig. 6a). The cluster-2 DMRs showed motif enrichment for IRF3 (interferon response), CEBPB (CEBPB/AP1 complex; immune response), EGR2 (cell proliferation, neural development), SOX2 (pluripotency), and TFAP4 (cell proliferation, differentiation blocking) (Fig. 6a). Many of these transcription factors were expressed in P0.5 PSGs or P7.5 SGs (Fig. 6b; Additional file 2: Figure S7). The cluster-1 and -2 DMRs associated with Stra8, Plzf, Smoc2, or Ski (Fig. 5) contained these motifs multiple times, as well as those similar to the binding motifs for SOX10 and SOX3 (Additional file 2: Figure S8). Although the significance of these motifs and transcription factors in SSC function and/or spermatogenesis awaits further studies, our results show that local methylation changes can identify likely candidates for regulatory regions.

\section{Discussion}

The neonatal and early postnatal stages are important for the establishment of the SSC system in the male germline. It is well established that epigenetic mechanisms play crucial roles in mammalian germline development [16], but how they contribute to SSC derivation and differentiation has not been addressed yet. In this study, we have performed WGBS and RNA-seq on neonatal PSGs and early postnatal SGs including the nascent SSC population and compared the results with those from fetal PSGs and adult spermatozoa. Below, we discuss our major findings with reference to the recently published WGBS and RNA-seq data from adult germ cells [24].

First, we identified PMDs in these germ cells, which are similar to those found in cultured human fibroblasts $[33,34]$, human cancer cells $[35,36]$, and human placenta [37]. The germline PMDs share features with those identified in other cell types: localization in genomic regions with low GC contents, low CGI density, low gene density, and overlap with cLADs. Furthermore, the genes in the PMDs were silenced, just as were those found in cancer cells $[35,36]$, sharing functional features. Since the PMDs in cancer cells are marked by repressive histone modifications such as $\mathrm{H} 3 \mathrm{~K} 27 \mathrm{me} 3$ and $\mathrm{H} 3 \mathrm{~K} 9 \mathrm{me} 3$ [36], genes located in the regions may be silenced through these modifications. We speculate that a similar silencing mechanism may operate in the germline PMDs because they share genomic localizations and structural features with the cancer PMDs. The PMDs were not seen in E10.5 primordial germ cells, but arose during the early phase of global de novo methylation in fetal PSGs, and persisted to the adult sperm stage. We confirmed the existence of PMDs in adult male germ cells, including the SSC-rich population $\left(\mathrm{Thy}^{+}{ }^{+}\right.$), using published data [21]. The mechanisms of PMD formation and the biological significance of PMDs are yet to be determined, but considering their association with cLADs, we speculate that germ cells, cancer cells, and placenta may have something in common in their nuclear architecture and/ or function.

Second, we found high levels of $\mathrm{CH}$ methylation and $5 \mathrm{hmCs}$ in neonatal PSGs. The $\mathrm{CH}$ methylation occurred globally, but predominantly in non-PMD regions, during the wave of de novo methylation in fetal PSGs arrested at $\mathrm{G} 1 / \mathrm{G} 0$. $\mathrm{CH}$ methylation reached the highest level in neonatal PSGs and disappeared after the resumption of mitosis. This confirmed and extended the previously observed $\mathrm{CH}$ methylation dynamics at SINE B1 copies [42]. Although we do not know the biological significance of this transient $\mathrm{CH}$ methylation, its accumulation is likely associated with the high de novo methylation activity and arrested cell cycle state of PSGs, as discussed previously [27]. So far, $\mathrm{CH}$ methylation has been reported in embryonic stem cells, brain tissues, and oocytes [27, 33, 65], but neonatal PSGs showed the highest level. In fact, two-thirds of all $5 \mathrm{mCs}$ of this cell type occurred at $\mathrm{CH}$ sites. The extremely high level of $\mathrm{CH}$ methylation and subsequent loss in early SGs help explain previous immunocytological observations, including the replication-dependent loss of $5 \mathrm{mC}$ signals [22]. In contrast to the $\mathrm{CH}$ methylation dynamics, CG methylation showed less changes (except for the stage-specific DMRs) during the neonatal and early postnatal stages. In particular, we confirmed the stable maintenance of CG methylation at the paternally methylated ICRs and retrotransposons. Thus, our study resolved the question previously posed by immunocytology regarding the maintenance of epigenetic imprints. As for $5 \mathrm{hmC}$, we confirmed previous immunocytological observations that chromosome satellite repeats are enriched in $5 \mathrm{hmC}$ in neonatal PSGs $[45,46]$ (Additional file 2: Figure S4). Interestingly, certain retrotransposons, such as IAP, L1MdA, and L1MdT, which are resistant to CG methylation reprogramming (demethylation) in primordial germ cells, had high levels of $5 \mathrm{hmC}$ and low levels of $\mathrm{CH}$ methylation in neonatal PSGs. This suggests that reprogramming-resistant regions accumulate $5 \mathrm{hmC}$ in primordial germ cells but do not undergo complete demethylation beyond this intermediate stage. Also, the de novo DNMTs responsible for $\mathrm{CH}$ methylation introduce few $\mathrm{CH}$ methylations in such reprogramming-resistant regions that maintain residual CG methylation. 
Third, we identified $>5000$ stage-specific DMRs showing developmental stage-specific changes $(>30 \%)$ in CG methylation between P0.5 PSGs and P7.5 SGs or between P7.5 $\mathrm{Kit}^{-}$and $\mathrm{Kit}^{+}$SGs, despite the small differences $(<0.6 \%)$ in global CG methylation. We focused on the 2800 DMRs that showed reduced methylation in P7.5 $\mathrm{Kit}^{-}$or $\mathrm{Kit}^{+}$SGs (cluster-1 and -2 DMRs) and found that many of them are located in intergenic regions or introns (not promoters), have histone marks specific for enhancers, and show association with specific gene functions such as cell proliferation, cell movement, stem cell function, and spermatogenesis. Such limited usage of differential promoter methylation for gene regulation has been observed in other developmental contexts, such as the lineage specification of human embryonic stem cells [66], highlighting that this phenomenon is not specific to male germ cell development. Approximately 700 DMR-linked genes showed expression changes ( $>2.0$-fold) between the stages, implying that the DMRs are indeed regulatory regions. The fact that most developmentally regulated DMRs map to distant cis regulatory regions rather than promoters fits well with the general landscape where DNA methylation is preferentially established in intergenic regions in male germ cells, while gene bodies and some CG-rich promoters are the favorite targets of methylation in female germ cells [28, 67]. Interestingly, most DMRs of clusters 1 and 2 stayed hypomethylated in the subsequent stages of spermatogenesis irrespective of gene activity, leaving the epigenetic signatures (hypomethylation) in the sperm genome. In early postnatal stages, de novo methylation occurred mainly in the PMDs and the DMRs of clusters 5 and 6, most of which were mapped in the PMDs. In this period, Dnmt3l was strongly downregulated (Additional file 2: Figure S1), suggesting that the de novo methylation is independent of DNMT3L. It is also possible that there may be a switch from DNMT3A to DNMT3B during this period, considering the changes in mRNA levels. However, targeted disruption of Dnmt3b in the male germline does not cause any phenotype [21], which seems consistent with the fact that the genes in the PMDs were consistently silent. A previous study on undifferentiated and differentiating adult SGs showed that methylation differences at promoters are rare (only seven promoters showed changes) [24]. We found only 10 promoter DMRs using the same criteria, confirming these observations in adult SGs. However, none of the seven promoters identified in the previous adult SSC study [24] coincided with our DMRs, perhaps because of the differences in developmental stage (adult versus early postnatal stage), cell markers used (Thy ${ }^{+}$and $\mathrm{Kit}^{-}$for the SSC-enriched population), or both.

Finally, the identified DMRs were extremely rich in binding motifs for specific transcription factors such as the SOX family members. The SOX family transcription factors are well-established regulators of cell fate decisions during development, with additional roles in adult tissue homeostasis and regeneration [68]. The role of SOX3 in SG differentiation has been established [62], but other SOX members expressed in early postnatal SGs might also play roles in SSC formation and differentiation. In addition to the SOX binding motifs, we identified potential binding motifs for many other transcription factors, some of which have a role in development or cell proliferation. It will be interesting to explore the role of these and related factors in SSC formation and/or differentiation.

\section{Conclusions}

Our WGBS and RNA-seq analyses revealed the existence of PMDs, a unique accumulation and distribution of $\mathrm{CH}$ methylation and $5 \mathrm{hmC}$ marks in neonatal PSGs, and stage-specific DMRs rich in specific transcription factor binding motifs. These findings contrast with the reported scarcity of differential methylation in adult SSCs differentiation and represent a unique phase of male germ cell development. Although the roles of the identified motifs and transcription factors in SSC function and/or spermatogenesis await further studies, our results show that local methylation changes can identify potential regulatory regions in developing germ cells. The data presented here will serve as an important resource for future studies, and our results provide important insights into the epigenetic regulation of SSC formation and differentiation.

\section{Methods}

Mouse husbandry

C57Bl/6 mice and Oct4-EGFP transgenic mice [11] maintained on a $\mathrm{C} 57 \mathrm{Bl} / 6$ background were housed and all experiments were performed under the ethical guidelines of Kyushu University, Yokohama City University, and Tokyo University of Agriculture.

\section{Preparation of germ cells}

PSGs and SGs were respectively isolated from P0.5 and P7.5 testes of Oct4-EGFP mice [11] by fluorescenceactivated cell sorting as described [69]. Briefly, testes were incubated in phosphate-buffered saline supplemented with $1 \mathrm{mg} / \mathrm{ml}$ collagenase (Sigma-Aldrich) and 100 units of DNase I (Invitrogen) for $15 \mathrm{~min}$ at $37{ }^{\circ} \mathrm{C}$ with agitation. For Kit staining, suspended cells were incubated at $4{ }^{\circ} \mathrm{C}$ with Fc-block for $15 \mathrm{~min}$ and then with an APC-anti-Kit antibody (eBiosciences) for $30 \mathrm{~min}$. Propidium iodide $(2 \mu \mathrm{g} / \mathrm{ml}$; Sigma-Aldrich) was added, and cells were sorted using MoFlo (Beckman-Coulter). The purity of the sample was checked by the expression of some marker genes (e.g., Oct4, Kit, and Plzf) using 
quantitative polymerase chain reaction. Isolation of E16.5 PSGs and adult spermatozoa was performed as previously described $[18,28]$.

\section{WGBS and analysis}

WGBS was done with two biological replicates for all cell types. DNA was extracted from $4-9 \times 10^{4}$ cells using the AllPrep DNA/RNA Mini Kit (Qiagen) according to the manufacturer's instructions. Libraries were prepared from $100 \mathrm{ng}$ of sample DNA spiked with $1 \mathrm{ng}$ of unmethylated lambda phage DNA (Promega) using the PBAT method [26, 27]. Concentrations of the PBAT products were quantified using the KAPA Illumina $\mathrm{Li}$ brary Quantification Kit (Kapa Biosystems). The libraries were sequenced on an Illumina HiSeq 2000 (HiSeq Control Software (HCS) version 1.5.15 and Real Time Analysis (RTA) version 1.13.48) or an Illumina HiSeq 2500 (HCS version 2.0.5 and RTA version 1.17.20) to generate 118-nucleotide (nt) single-end reads.

We obtained high-quality reads after trimming the low-quality bases from the $3^{\prime}$ end and the adapter sequences from the $5^{\prime}$ end using the NGS QC toolkit [70]. The resulting reads were aligned to the reference mouse genome (mm10) using Bismark alignment software (version 0.10.0) [71]. We used parameters of 28 for the seed length, 1 for the maximum number of mismatches permitted in the seed, and the option "-pbat" that works for PBAT libraries. Only uniquely aligned reads were analyzed. We estimated the bisulfite conversion rate using reads that were aligned uniquely to the lambda phage genome. Counts from the symmetric cytosines were combined for strand-independent analysis of CG methylation. We subsequently analyzed CG sites covered at least six times, $\mathrm{CH}$ sites covered at least four times, and discarded cytosines covered by more than 100 reads.

RefSeq genes and repeat element annotations (RepeatMasker) were downloaded from the UCSC genome browser database (mm10) [72]. Repeat consensus sequences were downloaded from Repbase [73]. The PMDs were identified using MethylSeekR [38].

To identify stage-specific DMRs, the methylation level of each $500 \mathrm{bp}$ window was determined from $\geq 5$ CG sites covered $\geq 10$ times. The window was identified as a DMR if the difference exceeded $30 \%$ and was shown significant by two-sample $t$-tests with unequal variance after correction for multiple hypotheses testing using the Benjamini-Hochberg method. Overlapping DMR windows (sliding steps of $100 \mathrm{bp}$ ) were merged. A cluster analysis of the identified DMRs was performed using $\mathrm{R}$ software with the complete linkage method (default). The CG methylation levels in the DMRs in P0.5 PSGs and the changes up to the P7.5 $\mathrm{Kit}^{+}$SG stage were used as the input dataset.

\section{Oxidative bisulfite sequencing and analysis}

Libraries were prepared from bisulfite-converted DNA and oxidized plus bisulfite-converted DNA, each from two biological replicates. Each library was prepared from 30 ng P0.5 PSG DNA with $0.3 \mathrm{ng}$ spike-in conversion controls. The DNA was sonicated to $500 \mathrm{bp}$ using the S220 Focused-ultrasonicator (Covaris) before spiking in. Oxidation reactions and bisulfite conversions were performed using TrueMethyl kits (Cambridge Epigenetix), and then oxidized and bisulfite-converted DNA or simply bisulfite-converted DNA was added with adapters using the PBAT method as described previously [26, 27]. The spike-in control was prepared using the protocol of $5 \mathrm{hmC}$ TAB-Seq kits (Wisegene). Trimmed high-quality reads were aligned to the reference mouse genome (mm10) or to the consensus major and minor satellite repeat sequences. The level of $5 \mathrm{hmC}$ was calculated by subtracting the value obtained from the oxidation plus bisulfite treatment dataset from that obtained from the corresponding bisulfite-only dataset.

\section{RNA-seq and analysis}

Two replicate preparations were analyzed for all cell types. Total RNA was extracted from $4-9 \times 10^{4}$ cells using the AllPrep DNA/RNA Mini Kit (Qiagen) or RNeasy Micro Kit (Qiagen) according to the manufacturer's instructions. Thirty to $100 \mathrm{ng}$ total RNA was subjected to enrichment for poly(A) ${ }^{+}$RNA and libraries were prepared using the TruSeq RNA Sample Prep Kit v2 (Illumina). For E16.5 PSGs, 10 ng of total RNA was used to synthesize and amplify double-stranded cDNAs using the SMARTer Ultra Low RNA kit (Clontech). The libraries were amplified for 15 cycles and then sequenced on an Illumina GAIIx instrument to generate 36-nt single-end reads or on an Illumina HiSeq 2000 instrument to generate 100-nt paired-end reads.

RNA-seq reads were mapped to the mouse mm10 genome assembly using TopHat (version 2.0.8) [74]. The mapped reads from two biological replicates were subsequently analyzed by Cufflinks (version 2.1.1) to estimate the transcript abundance [75], and the expression level of each transcript was indicated as a FPKM value.

\section{GO analysis}

The GO enrichment for stage-specific DMRs was done using GREAT (version 2.0) [53]. The FDR cutoff was 0.05 and the fold enrichment was kept at 2 . Genes linked to the DMRs were selected using basal plus extension criteria with a default setting (proximal, from $5 \mathrm{~kb}$ upstream to $1 \mathrm{~kb}$ downstream of the transcription start site; distal, up to $1 \mathrm{Mb}$ from the transcription start site), assuming that the DMRs were either proximal or distal regulatory regions. The GO analysis for differentially 
expressed genes was done using DAVID [54] with an allmouse gene background and the FDR cutoff at 0.1.

\section{Motif analysis}

We searched for de novo motifs enriched in each DMR cluster using the HOMER tool [61]. We used default parameters with a fragment size of 200 bp and with "-mask" parameter to use the repeat-masked sequences. Random background regions that matched the GCcontent distribution of the input DMR sequences were used as controls. The motif search for each DMR sequence was performed using the position weight matrix file of de novo motifs or known motifs from the motif database included in the HOMER tool with a relative profile score threshold $>90 \%$.

\section{Immunostaining}

For $5 \mathrm{mC}$ and $5 \mathrm{hmC}$ immunofluorescence staining, chromosomes fixed on slides were incubated in $4 \mathrm{~N} \mathrm{HCl}$ at $37^{\circ} \mathrm{C}$ for $30 \mathrm{~min}$. After neutralization in $100 \mathrm{mM}$ Tris$\mathrm{HCl}(\mathrm{pH} \mathrm{7.5)}$ for $10 \mathrm{~min}$, the specimens were incubated with anti-5mC (mouse monoclonal; Calbiochem) and/or anti-5hmC (rabbit polyclonal; Active Motif) antibodies. After blocking with $1 \%$ bovine serum albumin and $0.2 \%$ Triton X-100 in phosphate-buffered saline, the specimens were incubated with anti-mouse and/or antirabbit secondary antibodies coupled with Alexa Flour 488 or 594 (Life Technologies) at room temperature for $30 \mathrm{~min}$. The samples were then counterstained with DAPI in the mounting medium.

\section{Data from other sources}

Previously published WGBS data from mouse E13.5 primordial germ cells and E16.5 PSGs [18] and RNA-seq data from mouse adult spermatozoa [28] were downloaded and remapped to allow comparison with the current data. CGI data were taken from Illingworth et al. [76]. Previously published H3K27ac and H3K4me1 ChIP-seq data from the adult mouse testis [50] and cLAD data [77] were downloaded from the NCBI Gene Expression Omnibus database.

\section{Data access}

Sequence data have been deposited in DDBJ/GenBank/ EMBL under accession numbers DRA002477 and DRA002402.

\section{Additional files}

Additional file 1: Table S1. Sequencing runs and mapping summary. (XLSX $47 \mathrm{~kb}$ )

Additional file 2: Figures S1-S8. Figure S1: Expression of key molecular markers in isolated cells. Figure S2: CG methylation at ICRs and retrotransposons. Figure S3: PMD distribution in each chromosome.
Figure S4: Replication-dependent loss of $5 \mathrm{mC}$ and $5 \mathrm{hmC}$ in neonatal spermatogonia after the resumption of mitosis. Figure S5: Changes in gene expression levels between stages. Figure S6: Genes involved in the signal transduction pathways for SSC self-renewal have stage-specific DMRs. Figure S7: Expression dynamics of transcription factors listed by motif analysis of the DMRs. Figure S8: Motifs found in the representative DMRs. (PDF $26886 \mathrm{~kb}$ )

Additional file 3: Table S2. Locations and methylation levels of the DMRs. (XLSX $437 \mathrm{~kb}$ )

Additional file 4: Table S3. GO terms and genes obtained by the GREAT analysis. (XLSX $33 \mathrm{~kb}$ )

Additional file 5: Table S4. Expression levels of the differentially expressed genes. (XLSX $278 \mathrm{~kb}$ )

Additional file 6: Table S5. GO terms enriched for the differentially expressed genes identified by DAVID analysis. (XLSX 78 kb)

\section{Abbreviations}

5mC: 5-methylcytosine; 5hmC: 5-hydroxymethylcytosine; CGl: CG island; DMR: Differentially methylated region; EGFP: Enhanced green fluorescent protein; FPKM: Fragments per kilobase of exon per million mapped fragments; GO: Gene ontology; IAP: Intracisternal A particle; ICR: Imprinting control region; LINE: Long interspersed nuclear element; LTR: Long terminal repeat; PBAT: Post-bisulfite adaptor tagging; PGC: Primordial germ cell; PMD: Partially methylated domain; Pol2: RNA polymerase II;

PSG: Prospermatogonia; SINE: Short interspersed element;

SG: Spermatogonia; SSC: Spermatogonial stem cell; WGBS: Whole-genome bisulfite sequencing.

\section{Competing interests}

The authors declare that they have no competing interests.

\section{Authors' contributions}

KO and H. Sasaki: overall design. NK, T. Shirakawa, HK, H. Sone, Y. Sato, ST, TK, and $\mathrm{KO}$ : acquisition of samples. NK, KS, HK, and TK: experiments. YT, H. Shibata, H. Saitsu, Y. Suzuki, and NM: sequencing. NK, HT, T. Sato, and MS: data analysis and figures. NK and H. Sasaki wrote the manuscript. All authors read and approved the final manuscript.

\section{Acknowledgments}

We would like to thank Miho Miyake, Tomomi Akinaga, Junko Oishi (Kyushu University), Ikue Hoshi, Tamaki Nagasaka, Yoshito Kamizato, Nobuko Watanabe (Yokohama City University), Terumi Horiuchi (University of Tokyo), and Satoshi Sano (Tokyo University of Agriculture) for technical assistance and Illumina sequencing. We also thank Yoichi Nakanishi (Kyushu University) for generous support. K.S. is a Japan Society for the Promotion of Science (JSPS) research fellow. This work was supported in part by Grants-in-Aid for Scientific Research on Innovative Areas from the Ministry of Education, Culture, Sports, Science, and Technology of Japan to H. Sasaki (25112010), T.K. (22228004, 221S0002, and S0801025), and N.M. (24118007), and by the Core Research for Evolutional Science and Technology (CREST) from the Japan Agency for Medical Research and Development (AMED) to H. Sasaki. The work also received supports from a grant from AMED to N.M. (14525125) and the Kyushu University Interdisciplinary Programs in Education and Projects in Research Development (P\&P) to H. Sasaki.

\section{Author details}

${ }^{1}$ Division of Epigenomics and Development, Medical Institute of Bioregulation, Kyushu University, Fukuoka 812-8582, Japan. ${ }^{2}$ Research Institute for Disease of the Chest, Graduate School of Medical Sciences, Kyushu University, Fukuoka 812-8582, Japan. ${ }^{3}$ Department of Histology and Cell Biology, Yokohama City University School of Medicine, Yokohama 236-0004, Japan. ${ }^{4}$ NODAI Genome Research Center, Tokyo University of Agriculture, Tokyo 156-8502, Japan. ${ }^{5}$ Division of Bioinformatics, Medical Institute of Bioregulation, Kyushu University, Fukuoka 812-8582, Japan. ${ }^{6}$ Department of Human Genetics, Graduate School of Medicine, Yokohama City University, Yokohama 236-0004, Japan. ${ }^{7}$ Division of Genomics, Medical Institute of Bioregulation, Kyushu University, Fukuoka 812-8582, Japan. ${ }^{8}$ Department of Medical Genome Sciences, Graduate School of Frontier Sciences, The University of Tokyo, Kashiwanoha 5-1-5, Kashiwa, Chiba 277-8568, Japan. ${ }^{9}$ Department of BioScience, Tokyo University of Agriculture, Tokyo 156-8502, Japan. 
Received: 9 April 2015 Accepted: 7 August 2015

\section{Published online: 20 August 2015}

\section{References}

1. Brinster RL. Germline stem cell transplantation and transgenesis. Science. 2002;296:2174-6

2. Brinster RL, Avarbock MR. Germline transmission of donor haplotype following spermatogonial transplantation. Proc Natl Acad Sci U S A. 1994;91:11303-7.

3. Brinster RL, Zimmermann JW. Spermatogenesis following male germ-cell transplantation. Proc Natl Acad Sci U S A. 1994;91:11298-302.

4. Phillips BT, Gassei K, Orwig KE. Spermatogonial stem cell regulation and spermatogenesis. Philos Trans R Soc Lond B Biol Sci. 2010;365:1663-78.

5. Yoshida S, Sukeno M, Nakagawa T, Ohbo K, Nagamatsu G, Suda T, et al. The first round of mouse spermatogenesis is a distinctive program that lacks the self-renewing spermatogonia stage. Development. 2006;133:1495-505.

6. McLean DJ, Friel PJ, Johnston DS, Griswold MD. Characterization of spermatogonial stem cell maturation and differentiation in neonatal mice. Biol Reprod. 2003;69:2085-91.

7. Yoshida S, Takakura A, Ohbo K, Abe K, Wakabayashi J, Yamamoto M, et al. Neurogenin3 delineates the earliest stages of spermatogenesis in the mouse testis. Dev Biol. 2004;269:447-58.

8. Buaas FW, Kirsh AL, Sharma M, McLean DJ, Morris JL, Griswold MD, et al. Plzf is required in adult male germ cells for stem cell self-renewal. Nat Genet. 2004;36:647-52.

9. Costoya JA, Hobbs RM, Barna M, Cattoretti G, Manova K, Sukhwani M, et al. Essential role of Plzf in maintenance of spermatogonial stem cells. Nat Genet. 2004;36:653-9.

10. Meng X, Lindahl M, Hyvönen ME, Parvinen M, de Rooij DG, Hess MW, et al. Regulation of cell fate decision of undifferentiated spermatogonia by GDNF. Science. 2000;287:1489-93.

11. Ohbo K, Yoshida S, Ohmura M, Ohneda O, Ogawa T, Tsuchiya H, et al. Identification and characterization of stem cells in prepubertal spermatogenesis in mice. Dev Biol. 2003;258:209-25.

12. Sada A, Suzuki A, Suzuki H, Saga Y. The RNA-binding protein NANOS2 is required to maintain murine spermatogonial stem cells. Science. 2009;325:1394-8

13. Schrans-Stassen BH, van de Kant HJ, de Rooij DG, van Pelt AM. Differential expression of c-kit in mouse undifferentiated and differentiating type A spermatogonia. Endocrinology. 1999;140:5894-900.

14. Yoshinaga K, Nishikawa S, Ogawa M, Hayashi S, Kunisada T, Fujimoto T, et al. Role of c-kit in mouse spermatogenesis: identification of spermatogonia as a specific site of c-kit expression and function. Development. 1991;113:689-99.

15. Shinohara T, Orwig KE, Avarbock MR, Brinster RL. Spermatogonial stem cell enrichment by multiparameter selection of mouse testis cells. Proc Natl Acad Sci U S A. 2000;97:8346-51.

16. Sasaki H, Matsui Y. Epigenetic events in mammalian germ-cell development: reprogramming and beyond. Nat Rev Genet. 2008;9:129-40.

17. Kato Y, Kaneda M, Hata K, Kumaki K, Hisano M, Kohara Y, et al. Role of the Dnmt3 family in de novo methylation of imprinted and repetitive sequences during male germ cell development in the mouse. Hum Mol Genet. 2007;16:2272-80.

18. Kobayashi H, Sakurai T, Miura F, Imai M, Mochiduki K, Yanagisawa E, et al. High-resolution DNA methylome analysis of primordial germ cells identifies gender-specific reprogramming in mice. Genome Res. 2013;23:616-27.

19. Seisenberger S, Andrews S, Krueger F, Arand J, Walter J, Santos F, et al. The dynamics of genome-wide DNA methylation reprogramming in mouse primordial germ cells. Mol Cell. 2012;48:849-62.

20. Bourc'his D, Bestor TH. Meiotic catastrophe and retrotransposon reactivation in male germ cells lacking Dnmt3L. Nature. 2004:431:96-9.

21. Kaneda M, Okano M, Hata K, Sado T, Tsujimoto N, Li E, et al. Essential role for de novo DNA methyltransferase Dnmt3a in paternal and maternal imprinting. Nature. 2004;429:900-3.

22. Coffigny $\mathrm{H}$, Bourgeois C, Ricoul M, Bernardino J, Vilain A, Niveleau A, et al. Alterations of DNA methylation patterns in germ cells and Sertoli cells from developing mouse testis. Cytogenet Cell Genet. 1999;87:175-81.

23. Shirakawa T, Yaman-Deveci R, Tomizawa S, Kamizato Y, Nakajima K, Sone H, et al. An epigenetic switch is crucial for spermatogonia to exit the undifferentiated state toward a Kit-positive identity. Development. 2013;140:3565-76.
24. Hammoud SS, Low DH, Yi C, Carrell DT, Guccione E, Cairns BR. Chromatin and transcription transitions of mammalian adult germline stem cells and spermatogenesis. Cell Stem Cell. 2014;15:239-53.

25. de Rooij DG, Grootegoed JA. Spermatogonial stem cells. Curr Opin Cell Biol. 1998;10:694-701.

26. Miura F, Enomoto Y, Dairiki R, Ito T. Amplification-free whole-genome bisulfite sequencing by post-bisulfite adaptor tagging. Nucleic Acids Res. 2012;40:e136.

27. Shirane K, Toh H, Kobayashi H, Miura F, Chiba H, Ito T, et al. Mouse oocyte methylomes at base resolution reveal genome-wide accumulation of non-CpG methylation and role of DNA methyltransferases. PLoS Genet. 2013;9:e1003439

28. Kobayashi H, Sakurai T, Imai M, Takahashi N, Fukuda A, Yayoi O, et al. Contribution of intragenic DNA methylation in mouse gametic DNA methylomes to establish oocyte-specific heritable marks. PLoS Genet. 2012;8:e1002440.

29. Kanatsu-Shinohara M, Shinohara T. Spermatogonial stem cell self-renewal and development. Annu Rev Cell Dev Biol. 2013;29:163-87.

30. Ketola I, Rahman N, Toppari J, Bielinska M, Porter-Tinge SB, Tapanainen JS, et al. Expression and regulation of transcription factors GATA-4 and GATA-6 in developing mouse testis. Endocrinology. 1999;140:1470-80.

31. O'Shaughnessy PJ, Willerton L, Baker PJ. Changes in Leydig cell gene expression during development in the mouse. Biol Reprod. 2002;66:966-75.

32. Tomizawa S, Kobayashi H, Watanabe T, Andrews S, Hata K, Kelsey G, et al. Dynamic stage-specific changes in imprinted differentially methylated regions during early mammalian development and prevalence of non- $\mathrm{CpG}$ methylation in oocytes. Development. 2011;138:811-20.

33. Lister R, Pelizzola M, Dowen RH, Hawkins RD, Hon G, Tonti-Filippini J, et al. Human DNA methylomes at base resolution show widespread epigenomic differences. Nature. 2009:462:315-22.

34. Lister R, Pelizzola M, Kida YS, Hawkins RD, Nery JR, Hon G, et al. Hotspots of aberrant epigenomic reprogramming in human induced pluripotent stem cells. Nature. 2011;471:68-73.

35. Berman BP, Weisenberger DJ, Aman JF, Hinoue T, Ramjan Z, Liu Y, et al. Regions of focal DNA hypermethylation and long-range hypomethylation in colorectal cancer coincide with nuclear lamina-associated domains. Nat Genet. 2012;44:40-6.

36. Hon GC, Hawkins RD, Caballero OL, Lo C, Lister R, Pelizzola M, et al. Global DNA hypomethylation coupled to repressive chromatin domain formation and gene silencing in breast cancer. Genome Res. 2012;22:246-58.

37. Schroeder DI, Blair JD, Lott P, Yu HO, Hong D, Crary F, et al. The human placenta methylome. Proc Natl Acad Sci U S A. 2013;110:6037-42.

38. Burger L, Gaidatzis D, Schübeler D, Stadler MB. Identification of active regulatory regions from DNA methylation data. Nucleic Acids Res. 2013;41:e155.

39. Meuleman W, Peric-Hupkes D, Kind J, Beaudry JB, Pagie L, Kellis M, et al. Constitutive nuclear lamina-genome interactions are highly conserved and associated with A/T-rich sequence. Genome Res. 2013;23:270-80.

40. Oakes CC, La Salle S, Smiraglia DJ, Robaire B, Trasler JM. A unique configuration of genome-wide DNA methylation patterns in the testis. Proc Natl Acad Sci U S A. 2007;104:228-33.

41. Ikeda R, Shiura H, Numata K, Sugimoto M, Kondo M, Mise N, et al. Large, male germ cell-specific hypomethylated DNA domains with unique genomic and epigenomic features on the mouse $X$ chromosome. DNA Res. 2013;20:549-65.

42. Ichiyanagi T, Ichiyanagi K, Miyake M, Sasaki H. Accumulation and loss of asymmetric non- $\mathrm{CpG}$ methylation during male germ-cell development. Nucleic Acids Res. 2013:41:738-45.

43. Branco MR, Ficz G, Reik W. Uncovering the role of 5-hydroxymethylcytosine in the epigenome. Nat Rev Genet. 2012;13:7-13.

44. Gan H, Wen L, Liao S, Lin X, Ma T, Liu J, et al. Dynamics of 5hydroxymethylcytosine during mouse spermatogenesis. Nat Commun. 2013;4:1995.

45. Li Y, Miyanari Y, Shirane K, Nitta H, Kubota T, Ohashi H, et al. Sequence-specific microscopic visualization of DNA methylation status at satellite repeats in individual cell nuclei and chromosomes. Nucleic Acids Res. 2013;41:e186.

46. Yamaguchi S, Hong K, Liu R, Inoue A, Shen L, Zhang K, et al. Dynamics of 5methylcytosine and 5-hydroxymethylcytosine during germ cell reprogramming. Cell Res. 2013;23:329-39.

47. Booth MJ, Branco MR, Ficz G, Oxley D, Krueger F, Reik W, et al. Quantitative sequencing of 5-methylcytosine and 5-hydroxymethylcytosine at singlebase resolution. Science. 2012;336:934-7. 
48. Yu M, Hon GC, Szulwach KE, Song CX, Zhang L, Kim A, et al. Base-resolution analysis of 5-hydroxymethylcytosine in the mammalian genome. Cell. 2012;149:1368-80.

49. Stadler MB, Murr R, Burger L, Ivanek R, Lienert F, Schöler A, et al. DNAbinding factors shape the mouse methylome at distal regulatory regions. Nature. 2011:480:490-5.

50. Shen Y, Yue F, McCleary DF, Ye Z, Edsall L, Kuan S, et al. A map of the cis-regulatory sequences in the mouse genome. Nature. 2012;488:116-20.

51. Heintzman ND, Hon GC, Hawkins RD, Kheradpour P, Stark A, Harp LF, et al. Histone modifications at human enhancers reflect global cell-type-specific gene expression. Nature. 2009;459:108-12.

52. Heintzman ND, Stuart RK, Hon G, Fu Y, Ching CW, Hawkins RD, et al. Distinct and predictive chromatin signatures of transcriptional promoters and enhancers in the human genome. Nat Genet. 2007;39:311-8.

53. McLean CY, Bristor D, Hiller M, Clarke SL, Schaar BT, Lowe CB, et al. GREAT improves functional interpretation of cis-regulatory regions. Nat Biotechnol. 2010;28:495-501.

54. Huang DW, Sherman BT, Lempicki RA. Systematic and integrative analysis of large gene lists using DAVID bioinformatics resources. Nat Protoc. 2009;4:44-57.

55. Baltus AE, Menke DB, Hu YC, Goodheart ML, Carpenter AE, de Rooij DG, et al. In germ cells of mouse embryonic ovaries, the decision to enter meiosis precedes premeiotic DNA replication. Nat Genet. 2006;38:1430-4.

56. Koubova J, Menke DB, Zhou Q, Capel B, Griswold MD, Page DC. Retinoic acid regulates sex-specific timing of meiotic initiation in mice. Proc Natl Acad Sci U S A. 2006;103:2474-9.

57. Rocnik EF, Liu P, Sato K, Walsh K, Vaziri C. The novel SPARC family member SMOC-2 potentiates angiogenic growth factor activity. J Biol Chem. 2006;281:22855-64.

58. Barker $\mathrm{N}$, van Oudenaarden A, Clevers $\mathrm{H}$. Identifying the stem cell of the intestinal crypt: strategies and pitfalls. Cell Stem Cell. 2012;11:452-60.

59. Deheuninck J, Luo K. Ski and SnoN, potent negative regulators of TGF-beta signaling. Cell Res. 2009;19:47-57.

60. Liao HF, Chen WS, Chen YH, Kao TH, Tseng YT, Lee CY, et al. DNMT3L promotes quiescence in postnatal spermatogonial progenitor cells. Development. 2014;141:2402-13.

61. Heinz S, Benner C, Spann N, Bertolino E, Lin YC, Laslo P, et al. Simple combinations of lineage-determining transcription factors prime cis-regulatory elements required for macrophage and B cell identities. Mol Cell. 2010;38:576-89.

62. Raverot G, Weiss J, Park SY, Hurley L, Jameson JL. Sox3 expression in undifferentiated spermatogonia is required for the progression of spermatogenesis. Dev Biol. 2005;283:215-25.

63. Bondurand $\mathrm{N}$, Sham MH. The role of SOX10 during enteric nervous system development. Dev Biol. 2013;382:330-43.

64. Harris ML, Buac K, Shakhova O, Hakami RM, Wegner M, Sommer L, et al. A dual role for SOX10 in the maintenance of the postnatal melanocyte lineage and the differentiation of melanocyte stem cell progenitors. PLoS Genet. 2013;9:e1003644.

65. Xie W, Barr CL, Kim A, Yue F, Lee AY, Eubanks J, et al. Base-resolution analyses of sequence and parent-of-origin dependent DNA methylation in the mouse genome. Cell. 2012;148:816-31.

66. Gifford CA, Ziller MJ, Gu H, Trapnell C, Donaghey J, Tsankov A, et al. Transcriptional and epigenetic dynamics during specification of human embryonic stem cells. Cell. 2013;153:1149-63.

67. Smallwood SA, Tomizawa S, Krueger F, Ruf N, Carli N, Segonds-Pichon A, et al. Dynamic CpG island methylation landscape in oocytes and preimplantation embryos. Nat Genet. 2011;43:811-4.

68. Sarkar A, Hochedlinger K. The sox family of transcription factors: versatile regulators of stem and progenitor cell fate. Cell Stem Cell. 2013;12:15-30

69. Ohmura M, Yoshida S, Ide Y, Nagamatsu G, Suda T, Ohbo K. Spatial analysis of germ stem cell development in Oct-4/EGFP transgenic mice. Arch Histol Cytol. 2004;67:285-96.

70. Patel RK, Jain M. NGS QC Toolkit: a toolkit for quality control of next generation sequencing data. PLoS One. 2012;7:e30619.

71. Krueger F, Andrews SR. Bismark: a flexible aligner and methylation caller for Bisulfite-Seq applications. Bioinformatics. 2011;27:1571-2.

72. Meyer LR, Zweig AS, Hinrichs AS, Karolchik D, Kuhn RM, Wong M, et al. The UCSC Genome Browser database: extensions and updates 2013. Nucleic Acids Res. 2013:41:D64-9.

73. Jurka J, Kapitonov W, Pavlicek A, Klonowski P, Kohany O, Walichiewicz J. Repbase Update, a database of eukaryotic repetitive elements. Cytogenet Genome Res. 2005;110:462-7.
74. Trapnell C, Pachter L, Salzberg SL. TopHat: discovering splice junctions with RNA-Seq. Bioinformatics. 2009;25:1105-11.

75. Trapnell C, Roberts A, Goff L, Pertea G, Kim D, Kelley DR, et al. Differential gene and transcript expression analysis of RNA-seq experiments with TopHat and Cufflinks. Nat Protoc. 2012;7:562-78.

76. Illingworth RS, Gruenewald-Schneider U, Webb S, Kerr AR, James KD, Turner $D J$, et al. Orphan CpG islands identify numerous conserved promoters in the mammalian genome. PLoS Genet. 2010;6:e1001134.

77. Peric-Hupkes D, Meuleman W, Pagie L, Bruggeman SW, Solovei I, Brugman W, et al. Molecular maps of the reorganization of genome-nuclear lamina interactions during differentiation. Mol Cell. 2010;38:603-13.

\section{Submit your next manuscript to BioMed Central and take full advantage of:}

- Convenient online submission

- Thorough peer review

- No space constraints or color figure charges

- Immediate publication on acceptance

- Inclusion in PubMed, CAS, Scopus and Google Scholar

- Research which is freely available for redistribution 